\title{
End-to-End Delay Differentiation of IP Traffic Aggregates using Priority Queuing Models
}

\author{
Pedro Sousa, Paulo Carvalho and Vasco Freitas \\ Universidade do Minho, Departamento de Informática, \\ 4710-059 Braga, Portugal \\ email: $\{$ pns,paulo,vf\}@uminho.pt
}

\begin{abstract}
This article evaluates the use of Priority Queuing (PQ) Models to achieve delay differentiation in networks operating under the Class of Services paradigm. Three $P Q$ variants are considered: the proportional model, the additive model and a novel hybrid schema based on the upper time limit model. Through appropriate queueing and scheduling, delay differentiation is first considered for a node, and then extended to an endto-end perspective. For each model, the corresponding delay differentiation bounds are determined and verified resorting to simulation.
\end{abstract}

\section{Introduction}

The advent of Quality of Service (QoS) [1] in the Internet is being fostered by a crescent need to provide adequate network services to a vast range of QoS-demanding applications. In the presence of distinct applications and traffic profiles, service providers need to differentiate customers so that an efficient and cost-effective network resources management can be achieved. This will also allow charging network services according to the agreed QoS level guarantees. In addition to admission control, reservation protocols (e.g. RSVP [2]), resource management solutions [3, 4], or even when these are not present, acceptable QoS conditions can be obtained in the presence of an appropriate delay differentiation mechanism. In fact, delay differentiation can be extremely useful to integrate real-time applications (e.g. adaptive or tolerant $[5,6,7]$ ) and other delay sensitive applications [8]. Both proportional and additive models have been studied before $[9,10,11]$ as efficient forms to assure a delay differentiation between traffic classes. These models along with a more strict schema called Upper Time Limit model and a novel hybrid differentiation mechanism are also studied in [12]. This article evaluates the end-to-end differentiation capabilities of those models, establishing the corresponding upper bound equations for delay.
The mechanisms studied and the results obtained can be applied to models aiming the QoS provision in IP networks, such as the Differentiated Services approach [13].

\section{PQ Models}

Proportional, Additive and Upper Time Limit models belong to PQ Models [14]. In PQ models each queue is ruled by a priority function that varies over time (Time-Dependent Priorities). The nature of the priority function and its configuration parameters define the service discipline of each queue. This section reviews the three models briefly (see Fig. 1). This study considers $N$ distinct classes $C_{i(0 \leq i \leq N-1)}$ having $C_{0}$ the highest priority.

Proportional Model: Let $p_{i}(t)$ be the priority function associated with the queue $i$ and $U_{i}$ the corresponding differentiation parameter. In the proportional model this function is given by (1), with $t_{0}$ denoting the arrival time of a packet to queue $i$ and $U_{0}>U_{1}>\ldots>U_{N-1}$. The expected behaviour of a scheduler operating under (1) is that, under heavy load conditions, (2) is valid for all classes $(0 \leq i, j \leq N-1)$, being $\bar{d}_{i}, \bar{d}_{j}$ the mean queueing delays of the classes $i$ and $j$. In other words, the proportional relations expected in the delays result from the proportionality in the differentiation parameters.

$$
\begin{aligned}
& p_{i}(t)=\left(t-t_{0}\right) * U_{i} \\
& \frac{U_{i}}{U_{j}} \approx \frac{\bar{d}_{j}}{\bar{d}_{i}}
\end{aligned}
$$

Additive Model: The additive model differentiates queues by an additive constant as expressed in (3), with $U_{0}>U_{1}>\ldots>U_{N-1}$. In this case, the priority difference between two packets remains constant over time. The interesting point in this model is to study the possibility of achieving additive differentiation in class delays as expressed by (4), which denotes that high priority classes may have a delay gain over low priority classes reflecting the difference between the 


\begin{tabular}{|c||c|c|}
\hline \hline Proportional & Additive & Upper Time \\
\hline \hline$p(t)$ & & \\
\hline
\end{tabular}

Figure 1: Delay differentiation models.

differentiation parameters. If this is true, this model is an effective solution to spread class delays by a predefined value.

$$
\begin{aligned}
& p_{i}(t)=\left(t-t_{0}\right)+U_{i} \\
& {\left[\bar{d}_{i}-\bar{d}_{j}\right] \approx\left[U_{j}-U_{i}\right] \quad(i>j)}
\end{aligned}
$$

Upper Time Limit Model: The Upper Time Limit model is more rigid than the additive and proportional models as it imposes a finite queueing delay (reflected by $\left.U_{i}\right)$ ). In this model, the lower the boundary time is, the higher the priority function slope will be. At the limit $\left(\left(t-t_{0}\right) \geq U_{i}\right)$ the server is forced $^{1}$ to dispatch the packet waiting service. This model provides the protection of high priority classes as packets remain in queue for a maximum value of $U_{i}$ (see eq.(5)), in which $U_{0}<U_{1}<\ldots<U_{N-1}$. This class is oriented to applications where a bound on delay is mandatory. Considering this model, our objective is to establish delay bounds for the high priority class and, simultaneously, achieve proportional differentiation between the other classes. This can be done by combining differentiation parameters conveniently as explained in $[12]$.

$$
p_{i}(t)=\left\{\begin{array}{l}
\frac{\left(t-t_{0}\right)}{U_{i}-t+t_{0}} \text { if } t<t_{0}+U_{i} \\
\infty \text { if } t \geq t_{0}+U_{i}
\end{array}\right.
$$

An example of a node behaving under the three differentiation models is presented in [12].

\section{Experimental Framework}

The experimental framework was implemented resorting to the Network Simulator (NS) platform [15]. Each differentiation mechanism, which determines the scheduler behaviour, was implemented from Queue Class inheritance. Fig. 2 shows the simulation model where on-off ${ }^{2}$, exponential and isochronous traffic sources are mapped to different classes (A,B and C) contending for a common link. Each class contributes evenly for

\footnotetext{
${ }^{1}$ When the high priority class becomes overloaded the waiting time limit can be exceeded.

${ }^{2}$ On-off periods follow a Pareto distribution with $\alpha=1.2$.
}

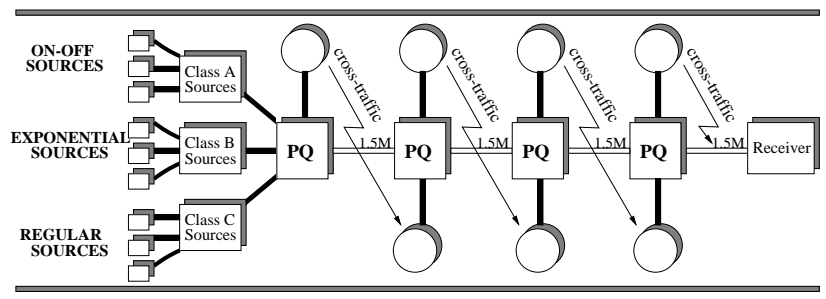

Figure 2: Simulation Scenario.

the overall load (i.e. long-term mean rates are similar) generating packets of 500 bytes. Similar queueing resources were also allocated for all classes. The end-to-end behaviour of the models is analysed for a scenario including four differentiation nodes traversed by the traffic aggregates defined. Additionally, several cross-traffic sources are considered in each differentiation node in order to improve the correctness of the simulation model. A modified version of an UDP receiver was also considered in order to calculate the end-to-end queueing delay ${ }^{3}$ of the traffic flows. As result, a scenario mixing different traffic patterns along a path including differentiation nodes under heavy load conditions was obtained ${ }^{4}$.

\section{End-to-end Differentiation}

\subsection{End-to-end Analysis}

This section studies the proportional, additive and upper time limit models from the end-to-end perspective, and establishes the corresponding upper bounds for delay differentiation. The definition of a differentiation domain aims to achieve a foreseeable relative differentiation behaviour for aggregate flows ${ }^{5}$ crossing a common set of nodes in a given time period. This domain consists of $M$ differentiation nodes traversed by individual flows. Let $\bar{d}_{i}^{j}$ be the average queueing delay of class $_{i}$ at node $j$. If a flow crosses $M$ servers $(0 \leq j \leq M-1)$, then the end-to-end average queueing delay $\left(\bar{d}_{i}^{*}\right)$ of class $_{i}$ can be expressed by (6). For additive differentiation and under heavy load, equation (7) can be applied to a generic server where $U_{i}^{j}$ is the differentiation parameter of class $_{i}$ in node $j$. Now, considering a flow crossing $M$ independent nodes, (7) becomes (8) which combined with equation (6) results in $(9)$.

$\bar{d}_{i}^{*}=\sum_{j=0}^{M-1} \bar{d}_{i}^{j}$

\footnotetext{
${ }^{3}$ This delay excludes propagation delay and packet transmission time.

${ }^{4}$ Obviously, due to traffic oscillations there are periods where the nodes are not heavily load.

${ }^{5}$ The differentiation behaviour is also kept at flow level [12].
} 
$\left(\bar{d}_{i+1}^{j}-\bar{d}_{i}^{j}\right) \approx\left(U_{i}^{j}-U_{i+1}^{j}\right) \Rightarrow \bar{d}_{i+1}^{j} \approx\left(U_{i}^{j}-U_{i+1}^{j}\right)+\bar{d}_{i}^{j}(7)$

$\sum_{j=0}^{M-1} \bar{d}_{i+1}^{j} \approx \sum_{j=0}^{M-1}\left(U_{i}^{j}-U_{i+1}^{j}\right)+\sum_{j=0}^{M-1} \bar{d}_{i}^{j}$

$\bar{d}_{i+1}^{*} \approx \sum_{j=0}^{M-1}\left(U_{i}^{j}-U_{i+1}^{j}\right)+\bar{d}_{i}^{*}$

Equation (9) is obtained considering all servers in the flows' path under heavy load conditions, otherwise the distance between class delays may become smaller. Equation (10) denotes this aspect and establishes an upper-bound for the end-to-end additive differentiation behaviour between adjacent classes. A constant $\epsilon$ needs to be introduced due to possible inaccuracies of the models when the average delays are measured in small time scales and, simultaneously, the server is suffering high class load oscillations.

$$
\left(\bar{d}_{i+1}^{*}-\bar{d}_{i}^{*}\right)<\sum_{j=0}^{M-1}\left(U_{i}^{j}-U_{i+1}^{j}\right)+\epsilon
$$

For the proportional model, formula (7) is now replaced by (11). Considering again a generic case of $M$ servers under heavy load, equation (11) becomes (12), where the right term of equation (12) can be expanded to $(13)$.

$$
\begin{aligned}
& \frac{\vec{d}_{i+1}^{j}}{\bar{d}_{i}^{j}} \approx \frac{U_{i}^{j}}{U_{i+1}^{j}} \Rightarrow \bar{d}_{i+1}^{j} \approx\left(\frac{U_{i}^{j}}{U_{i+1}^{j}}\right) * \bar{d}_{i}^{j} \\
& \sum_{j=0}^{M-1} \bar{d}_{i+1}^{j} \approx \sum_{j=0}^{M-1}\left[\left(\frac{U_{i}^{j}}{U_{i+1}^{j}}\right) * \bar{d}_{i}^{j}\right] \\
& \frac{U_{i}^{0}}{U_{i+1}^{0}} * \bar{d}_{i}^{0}+\frac{U_{i}^{1}}{U_{i+1}^{1}} * \bar{d}_{i}^{1}+\ldots+\frac{U_{i}^{M-1}}{U_{i+1}^{M-1}} * \bar{d}_{i}^{M-1}
\end{aligned}
$$

Defining now $X$ and $Y$ as (14.1) and (14.2), the equation (13) can be bounded by (15). Using the same arguments of the additive models and considering equations (6) (12) and (15), equation (16) establishes an upper bound for end-to-end proportional delay differentiation between adjacent traffic classes.

$$
\begin{aligned}
& X=\min _{0 \leq j \leq M-1}\left(\frac{U_{i}^{j}}{U_{i+1}^{j}}\right) \\
& Y=\max _{0 \leq j \leq M-1}\left(\frac{U_{i}^{j}}{U_{i+1}^{j}}\right) \\
& X *\left(\sum_{j=0}^{M-1} \bar{d}_{i}^{j}\right) \leq(13) \leq Y *\left(\sum_{j=0}^{M-1} \bar{d}_{i}^{j}\right) \\
& \left(\frac{\bar{d}_{i+1}^{*}}{d_{i}^{*}}\right)<\max _{0 \leq j \leq M-1}\left(\frac{U_{i}^{j}}{U_{i+1}^{j}}\right)+\epsilon
\end{aligned}
$$

For the upper time limit model a high priority class under feasible load conditions is expected to achieve a maximum queueing delay equivalent to the sum of the differentiation parameters of class $_{0}$ along the path, expressed by (17). Within the hybrid model presented in [12], low priority classes obtain an end-to-end relation also expressed by equation (16).

$$
\bar{d}_{0}^{*}=\sum_{j=0}^{M-1} \bar{d}_{0}^{j}<\sum_{j=0}^{M-1} U_{0}^{j}+\epsilon
$$

\subsection{Performance Evaluation}

The results presented in Fig. 3 and 4 show the endto-end queueing delay relations of each traffic class for each differentiation model. The results are obtained for intervals of $50 \mathrm{~ms}$ (Fig. 3(a) to (c) and Fig. 4(a)(b)) and for the full simulation period (Fig. 3(d) to (f) and Fig. 4(c)). Table 1 summarises the models characteristics discussed in this section.

Proportional Differentiation: Fig. 3(a)(d) shows the proportional relations between queueing delays obtained for a scenario when all differentiation nodes have $\left(U_{A}, U_{B}, U_{C}\right)=(4,2,1)$. The results show that end-toend proportional relations are achieved for the classes. In fact, as depicted in Fig. 3(a), for short-time scale, the ratio Class $_{C} /$ Class $_{B}$ is around 2 (which corresponds to $\left.\max \left(U_{B} / U_{C}\right)\right)$. The same reasoning can be made for the relation between Class $_{C} /$ Class $_{A}$ (with a ratio of 4) and, transitively for Class $_{B} /$ Class $_{A}$. Also note that there is a group of points above the expected ratios due to class load oscillations on the server and to the short-time scale used to measure queueing delay averages. This is sustained by the $\epsilon$ constant introduced in equation (16). As plotted in Fig. 3(a) such values show acceptable deviations from the desired value taking into account the fine grain of the measurements. As the time-scale increases the influence of such deviations decreases. Fig. 3(d) shows the average values for the delay ratio between the classes, as depicted and for most of the simulation periods, each delay ratio does not exceed the upper bound configuration ratio. This means that for large-time scales (in this case 10s) the significance of the constant $\epsilon$ decreases sharply. Although the equation (16) establishes an upper-bound for end-to-end proportional differentiation, it is important verify the sensibility of the equation to heterogeneous parameter configuration. To illustrate this aspect Fig. 3(b)(e) shows the differentiation results when a lower rate node is considered in the simulation scenario. All the previous considerations for Fig. 3(a)(d) are assumed, with the exception that one of the four differentiation nodes is now configured with $\left(U_{A}, U_{B}, U_{C}\right)=(3,2,1)$, and the outgoing link has a capacity of 0.5 instead $1.5 \mathrm{Mbps}$. As depicted in Fig. 3(b)(e), the delay ratio is now 3 for Class $_{C} /$ Class $_{A}$ and 1.5 for Class $C$ /Class $B$. This means that the slow rate differentiation node becomes more influent than before as regards the end-to-end differentiation behaviour. For this reason one can conclude that for heterogeneous parameters configuration, lower rate nodes can cause significant deviations from the upper bound expressed by equation (16).

Additive Differentiation: Fig. 3(c)(f) shows the additive relations between queueing delays obtained for a scenario where all differentiation nodes are configured 

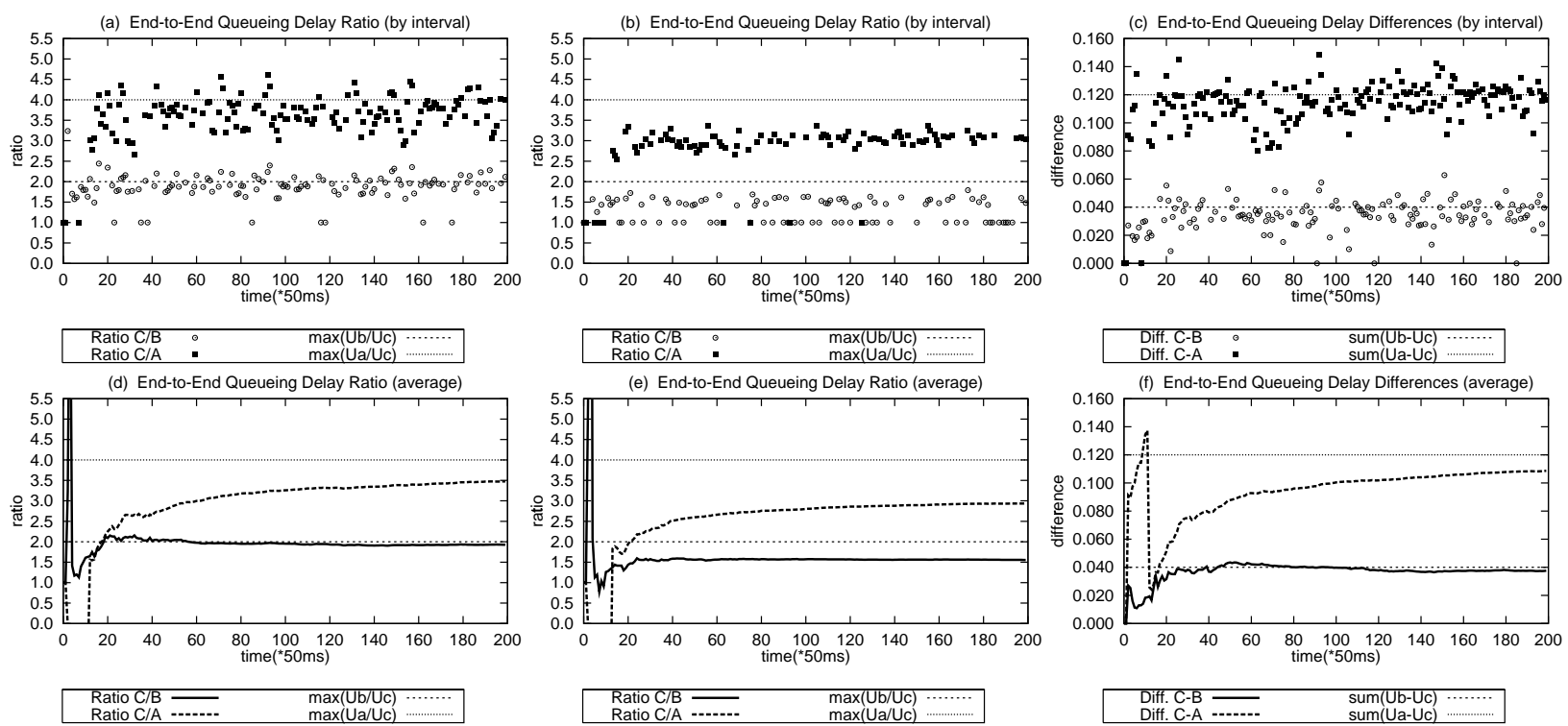

Figure 3: (a)(d) End-to-end Delay Ratio -Prop. Model- four nodes with $\left(U_{A}, U_{B}, U_{C}\right)=(4,2,1)$. (b)(e) Endto-end Delay Ratio -Prop. Model- three nodes with $\left(U_{A}, U_{B}, U_{C}\right)=(4,2,1)$ and a low rate node with $(3,2,1)$. (c)(f) End-to-end Delay Differences -Add. Model- four nodes with $\left(U_{A}, U_{B}, U_{C}\right)=(0.030,0.010,0.0)$.
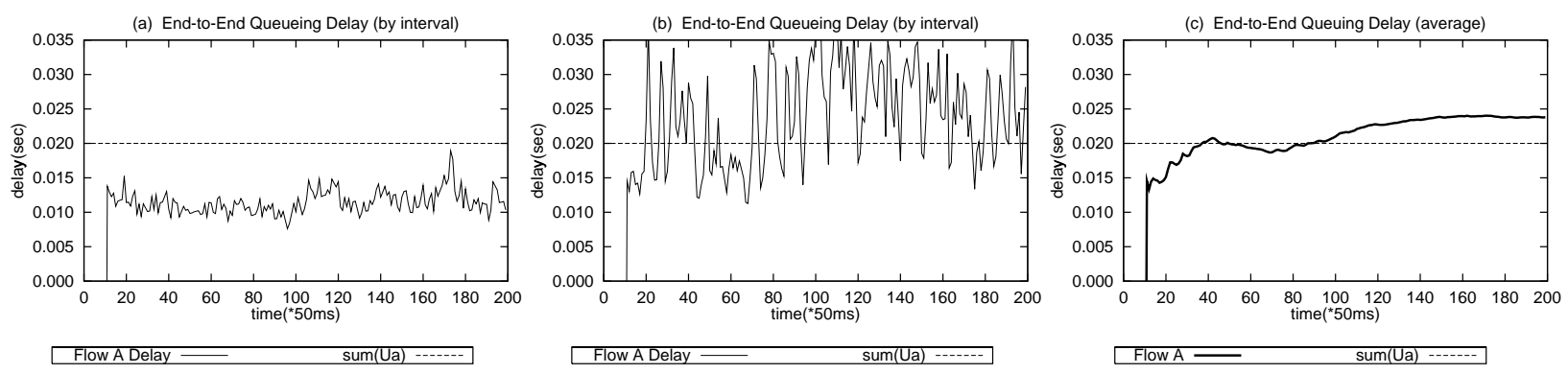

Figure 4: End-to-end delay for the Upper Time Limit Model: (a) four nodes with $\left(U_{A}, U_{B}, U_{C}\right)=(0.005,-,-)$. (b)(c) four nodes with $\left(U_{A}, U_{B}, U_{C}\right)=(0.005,-,-)$ and excessive load on Class $_{a}$.

Table 1: Models Characteristics. Legend: (-) Almost none (+/-) Low (+) High.

\begin{tabular}{|c|c|c|c|c|c|}
\hline \multirow[b]{2}{*}{ Model } & \multirow[b]{2}{*}{ Node Behaviour } & \multirow[b]{2}{*}{ End-to-End Behaviour } & \multirow[b]{2}{*}{ Comments } & \multicolumn{2}{|c|}{$\bar{\epsilon}$ significance } \\
\hline & & & & $\begin{array}{l}\text { Short } \\
\text { time } \\
\text { scale }\end{array}$ & $\begin{array}{l}\text { Large } \\
\text { time } \\
\text { scale }\end{array}$ \\
\hline Proportional & 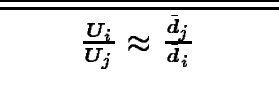 & $\left(\frac{\bar{d}_{i+1}^{*}}{d_{i}^{*}}\right)<\max _{0 \leq j \leq M-1}\left(\frac{U_{i}^{j}}{U_{i+1}^{j}}\right)+\epsilon$ & $\begin{array}{l}\text { Low rate nodes } \\
\text { influence }\end{array}$ & $(+/-)$ & $(-)$ \\
\hline Additive & $\bar{d}_{i}-\bar{d}_{j} \approx U_{j}-U_{i}$ & $\left(\bar{d}_{i+1}^{*}-\bar{d}_{i}^{*}\right)<\sum_{j=0}^{M-1}\left(U_{i}^{j}-U_{i+1}^{j}\right)+\epsilon$ & & $(+/-)$ & $(-)$ \\
\hline Upper Time & $\bar{d}_{0}<U_{0}$ & $\bar{d}_{0}^{*}<\sum_{j=0}^{M-1} U_{0}^{j}+\epsilon$ & $\begin{array}{c}\text { Feasibility } \\
\text { problems } \\
\text { due to no Class } s_{0} \\
\text { load control }\end{array}$ & $(+)$ & $(+)$ \\
\hline
\end{tabular}


with parameters $\left(U_{A}, U_{B}, U_{C}\right)=(0.030,0.010,0.0)$. The results show that end-to-end additive relations are obtained for the classes. In fact, as depicted in Fig. 3(c), for short-time scales, the queueing delay differences between Class $_{C}$ and Class $_{B}$ are around $40 \mathrm{~ms}$ (which corresponds to the sum of $U_{B}-U_{C}$ for all nodes in the path, i.e. $4 * 10 \mathrm{~ms}$ ). The same reasoning can be made for the relation between Class $_{C}$ and Class $_{A}$ (with a difference value of $120 \mathrm{~ms}$ ), and transitively for Class $_{B}$ and Class $_{A}$. As the proportional model, there is a group of points above the expected differences also sustained by the constant $\epsilon$ introduced in equation (10). Once again, the deviations from the desired differentiation values are acceptable. As in the proportional model, when the measurement scale increases the influence of such deviation gets smaller. Fig. 3(f) shows the average values for the delay differences between the classes, as depicted and for most of the simulation periods, the delay differences do not exceed the sum of the differences between the configuration parameters.

Upper Time Limit Model: As explained the upper time limit model is used as an hybrid mechanism, aiming to provide a queueing delay limit to $\mathrm{Class}_{A}$, and simultaneously, to achieve proportional differentiation between the other classes. The latter objective is achieved as for the proportional model. As regards the objective to limit queueing delays on Class $_{A}$, the results show that it heavily depends on the traffic aggregate in that class, i.e., Class $_{A}$ load must be confined to an upper bound value in order to not impair the configuration limit delay $\left(U_{A}\right)$ introduced in the differentiation node. Fig. 4 illustrates the Class $_{A}$ queueing delays when, for the high priority class, the configuration nodes are under a $5 \mathrm{~ms}$ delay limit $\left(U_{A}, U_{B}, U_{C}\right)=(0.005,-,-)$. Fig. 4(a) shows that end-to-end queueing is under the end-toend target bound $\left(4^{*} 5=20 \mathrm{~ms}\right)$. However, if Class $_{A}$ traffic aggregate becomes excessive (e.g. due the absence of admission control procedures), the end-to-end queueing delays of Class $_{A}$ become greater than the desired values (for short-time scales or on average, as Fig. 4(b)(c) shows). This means that the constant $\epsilon$ in equation (17) can become very significant to the endto-end model behaviour.

\section{Conclusions}

This paper assesses the use of PQ models in achieving end-to-end delay differentiation between distinct traffic classes. In particular, the proportional, additive and upper time limit models are considered and the work presented in [12] is extended by establishing the corresponding equations for end-to-end delay differen- tiation upper bounds. The accuracy of these equations is verified for short and large time measurement scales.

\section{References}

[1] G. Armitage. Quality of Service in IP Networks: Foundations for a Multi-Service Internet, Macmillan Technical Publishing, Apr. 2000.

[2] R. Braden et al. Resource Reservation Protocol (RSVP). RFC2205, Sep. 1997.

[3] I. Stoica and H. Zhang. Providing Guaranteed Services without Per Flow Management, In Proc. of SIGGCOMM'99, 1999.

[4] Z. Zhang et al. Decoupling QoS Control from Core Routers: A Novel Bandwidth Broker Architecture for Scalable Support of Guaranteed Services, In Proc. of SIGCOMM'00, 2000.

[5] P. Sousa and V. Freitas. A framework for the development of tolerant real-time applications, Computer Networks and ISDN Systems 30, 1531-1541, Dec. 1998.

[6] T. Nandagopal, N. Venkitaraman. Delay Differentiation and Adaptation in Core Stateless Networks, Proc. of INFOCOM 2000, Tel Aviv, Israel - Volume 2.

[7] I. Busse et al. Dynamic QoS Control of Multimedia Applications based on RTP, Computer Communications, Vol. 19, N. 1, pp. 49-58, Jan. 1996.

[8] M. Baldi. End-to-End Delay Analisys of VideoConferencing over Packet-Switched Networks, IEEE/ACM Trans. on Net., Vol. 8, N. 4, Aug. 2000.

[9] C. Dovrolis and P. Ramanathan. A Case for Relative Differentiated Services and the Proportional Differentiation Model, IEEE Network Magazine, 1999.

[10] C. Dovrolis and D. Stiliadis. Relative Differentiated Services in the Internet: Issues and Mechanisms, In Proc. of ACM SIGMETRICS'99.

[11] C. Dovrolis et al. Proportional Differentiated Services: Delay Differentiation and Packet Scheduling, In Proc. of ACM SIGCOMM'99, 1999.

[12] P. Sousa et al. Tuning Delay Differentiation in IP Networks using Priority Queueing Models, In Proc. of IFIP Networking2002 Conf., Pisa, Italy, May 2002.

[13] S. Blake el al. An Architecture for Differentiated Services, RFC2475, Dec. 1998.

[14] G. Bolch et al. Queueing Networks and Markov Chains - Modeling and Performance Evaluation with Computer Science Applications, John Wiley \& Sons INC., 1998.

[15] $n s$ Documentation. http://www.isi.edu/nsnam/ns/nsdocumentation.html 